\title{
SEOM clinical guideline thyroid cancer (2019)
}

\author{
E. Gallardo ${ }^{1}$ (I) J. Medina ${ }^{2} \cdot$ J. C. Sánchez ${ }^{3} \cdot$ A. Viúdez $^{4} \cdot$ E. Grande $^{5} \cdot$ I. Porras $^{6} \cdot$ T. Ramón y Cajal $^{7} \cdot$ J. Trigo $^{8}$. \\ L. Iglesias $^{9} \cdot$ J. Capdevila ${ }^{10}$
}

Received: 27 December 2019 / Accepted: 27 December 2019 / Published online: 31 January 2020

(c) The Author(s) 2020

\begin{abstract}
Thyroid carcinoma is the most frequent endocrine malignancy and accounts for around 3\% of global cancer incidence. Different histologies and clinical scenarios make necessary a multidisciplinary approach that includes new diagnostic methods and surgical, radiopharmaceutical and systemic therapies. This guideline updates several aspects of management of thyroid cancer.
\end{abstract}

Keywords Thyroid cancer $\cdot$ Multidisciplinary $\cdot$ Systemic therapy

\section{Methodology}

SEOM guidelines have been developed with the consensus of ten members from the Spanish Society of Medical Oncology (SEOM). In order to assess the level and quality of evidence and to establish a grade of recommendation of the different statements in this guideline, we based ourselves on The Infectious Diseases Society of America-US Public
Health Service Grading System (Table 1). The final text was reviewed and approved by all the authors. The goal of this document consists of providing clear practical recommendations about the management of thyroid cancer.

\section{E. Gallardo \\ egallardo@tauli.cat \\ J. Medina \\ boladiez39@yahoo.es \\ J. C. Sánchez \\ juancristobal.sg@gmail.com}

\section{A. Viúdez \\ aviudez@outlook.es \\ E. Grande \\ egrande@mdanderson.es}

I. Porras

iporras@wanadoo.es

T. Ramón y Cajal

tramon@santpau.cat

J. Trigo

jmtrigo@seom.org

L. Iglesias

laraiglesias@hotmail.com

J. Capdevila

jacapdevila@vhebron.net

1 Oncology Department, Parc Taulí Hospital Universitari, Institut d'Investigació i Innovació Parc Taulí I3PT,
Universitat Autònoma de Barcelona, 08208 Barcelona, Sabadell, Spain

2 Oncology Department, Hospital Virgen de la Salud, Toledo, Spain

3 Oncology Department, Hospital Universitario Puerta de Hierro, Majadahonda, Madrid, Spain

4 Oncology Department, Complejo Hospitalario de Navarra, Pamplona, Spain

5 Oncology Department, MD Anderson Cancer Center, Madrid, Spain

6 Oncology Department, Hospital Universitario Reina Sofía, Córdoba, Spain

7 Oncology Department, Hospital de la Santa Creu i Sant Pau, Barcelona, Spain

8 Oncology Department, Hospital Universitario Regional Virgen de la Victoria, Málaga, Spain

9 Oncology Department, Hospital Universitario 12 de Octubre, Madrid, Spain

10 Oncology Department, Hospital Universitari Vall d'Hebron, Vall d'Hebron Institut d'Oncologia (VHIO), Barcelona, Spain 
Table 1 Infectious diseases society of America-US Public Health Service Grading System

\begin{tabular}{ll}
\hline Category, grade & Definition \\
\hline Strength of recommendation & \\
A & Good evidence to support a recommendation for use \\
B & Moderate evidence to support a recommendation for use \\
C & Poor evidence to support a recommendation \\
D & Moderate evidence to support a recommendation against use \\
E & Good evidence to support a recommendation against use \\
Quality of evidence & \\
I & Evidence from $\geq 1$ properly randomized, controlled trial \\
II & Evidence from $\geq 1$ well-designed clinical trial, without rand- \\
& omization; from cohort or case-controlled analytic studies \\
& (preferably from $>1$ center); from multiple time series; or \\
& from dramatic results from uncontrolled experiments \\
III & Evidence from opinions of respected authorities, based \\
& on clinical experience, descriptive studies, or reports of \\
& expert committees \\
\hline
\end{tabular}

Note: From [43]

\section{Introduction}

Thyroid cancer is the most common endocrine malignancy and accounts for $3.1 \%$ of global cancer incidence. In 2018, 567,000 new cases were estimated worldwide, with differences by geographical area, age and sex. The global age-adjusted incidence rate in women is three times higher than in men: 10.2 and 3.1 per 100,000 , respectively [1]. The estimated incidence of thyroid cancer in Spain in 2018 was 13.7 per 100,000 in women and 3.8 per 100,000 in men [2]. A higher incidence is found in developed countries, compared to developing ones [1] and race-ethnic differences could be found [3]. However, despite the incidence differences, mortality rates are quite similar between countries, gender and approximately the same in all ethnic groups [3].

Incidence has increased progressively worldwide in recent decades, mostly secondary to small papillary carcinomas as the main consequence of a better diagnostic accuracy and overdiagnosis of indolent disease [4]. The only well established risk factor for thyroid cancer is ionizing radiation, particularly when exposure is in childhood, although there is evidence that other factors (obesity, smoking, hormonal exposures, and certain environmental pollutants) may play a role [1].

Based on the pathology, thyroid cancer can be classified into subtypes: differentiated, medullary, poorly differentiated and anaplastic carcinoma. Differentiated thyroid cancer (DTC), which arises from follicular thyroid cells, is the most common type and is subdivided into papillary thyroid carcinoma, $85 \%$ of thyroid cancer, follicular thyroid carcinoma, 2-5\%, and Hürthle-cell carcinomas accounting for $2 \%$ [5]. Certain subtypes of papillary cancer: tall cell variant, columnar cell variant, and diffuse sclerosing variant, have a worse prognosis, as do more highly invasive variants of follicular cancer. Medullary thyroid carcinoma, thyroid C-cell derived tumor, accounts for $4 \%$ of thyroid cancer. Poorly differentiated, $6 \%$, and anaplastic thyroid carcinomas, $1 \%$, are associated with aggressive disease [5].

Although most cases are sporadic, 3-9\% of DTC are familial, mainly component of cancer syndromes: Cowden's disease, familial adenomatous polyposis, and Werner's syndrome, are caused by germline loss-of function mutations in PTEN, $A P C$, and WRN5, respectively. $20 \%$ of medullary thyroid carcinoma are related to hereditary multiple endocrine neoplasia (MEN) type 2 syndromes, caused by autosomal dominant gain-of-function mutations in the RET proto-oncogene. There are three subtypes: MEN2A, MEN2B and familial medullary thyroid cancer (FMTC). 98\% of MEN2A, $99-100 \%$ of MEN2B, and 85\% of FMTC cases, present mutations in RET. $6 \%$ of patients with clinically sporadic medullary thyroid cancer carry a germline mutation in RET [5].

The majority of patients have an excellent prognosis, with a 5-year relative overall survival of $98.2 \%$ for all stages (99.9\% localized; 98.2\% regional; 56.2\% distant disease) [3] and, despite increasing incidence, mortality rates from the disease remain stable and low, with age-adjusted rates of 0.4 and 0.5 per 100,000 men and women [1].

\section{Diagnosis, pathology and molecular classification}

A radiological study by ultrasound (US) must be carried out in any patient with a thyroid gland alteration detected at physical examination, or with an anomalous/incidental 
nodular image observed by any imaging test $[6,7]$. Although around half of the population may have thyroid nodules, TC (thyroid cancer) diagnosis is rare (about 5\% of all thyroid nodules). Some US characteristics may increase the specificity for diagnosis beyond $90 \%$ (microcalcifications, hypoecogenicity, higher shape taller that longer, irregular margins, solid appearance, absence of halo, intranodular blood flow) [8]. In this sense, the American Thyroid Association (ATA) has stratified the US findings in five risk groups (from highly suspicious to clearly benign) to justify the performance of fine-needle-aspiration (FNA) [9]. Regardless of risk stratification, a FNA is recommended in any thyroid nodule $>1 \mathrm{~cm}$ or in those smaller but with clinical suspicion (previous head and neck irradiation, family history of TC, suspicion features at palpation and/or presence of cervical node). However, in case of low-risk echographic pattern, such as in cystic or spongiform nodules or isoechoic without halo or hyperechoic nodules, FNA is recommended when size is $>2 \mathrm{~cm}$. Due to early nodal dissemination ( $>90 \%$ of cases present at least microscopic nodal involvement in histopathological analysis), US may detect nodal involvement in more than $20 \%$ of cases [10]. Based on FNA findings, the 2017-version of Bethesda classification includes six scenarios (from non-diagnostic/unsatisfactory-Bethesda I to diagnosis of malignancy-Bethesda VI) with their respective risk of malignancy based on the inclusion or not of neoplasm with papillary-like nuclear features [11]. This updated classification also assesses the performance of molecular tests as well as their hypothetical role to further refine the estimation of malignancy of undetermined thyroid nodules. In this way, several molecular tests based on transcriptomic analysis, mutational panels (most of them focused on BRAF, HRAS, KRAS, PIK3CA and fusion genes such as PTC1, PTC3, $P A X 8 / P P A R \gamma, R E T, N T R K, A L K$, etc.) and microRNA classification are currently being developed, in spite of their real chance for routine clinical implementation should still overcome multiple methodological, availability and/or clinical effectiveness issues [12]. Routine preoperative thyroglobulin (Tg) is not recommended although measurement of serum calcitonin can be useful for medullary thyroid cancer (MTC) even with higher sensitivity than FNA in this context [8].

\section{Recommendations}

Patients with suspicion of thyroid alteration during the physical examination, or in which an anomalous/incidental nodular image is observed by any imaging test should undergo neck ultrasonography (level of evidence: I, grade of recommendation: A). It should be complemented with a FNA in any thyroid nodule with clinical suspicion. Routine preoperative serum biomarkers may be useful in MTC scenario (level of evidence: III, grade of recommendation: B).

\section{Differentiated thyroid cancer}

\section{Surgical treatment}

Most guidelines recommend total thyroidectomy in patients with nodules $>4 \mathrm{~cm}$ and who tolerate surgical treatment, since it is associated with greater disease-free survival, covers the possibility of multicentricity of papillary carcinoma (30-40\% of patients), it allows the use of radioactive iodine as a diagnostic and therapeutic tool and facilitates the monitoring of $\mathrm{Tg}$ (always together with autoantibodies against $\mathrm{Tg}, \mathrm{TgAb}$ ) as a marker of persistence or disease recurrence. It also reduces the need for second surgeries. For patients with thyroid cancer $>1 \mathrm{~cm}$ and $<4 \mathrm{~cm}$ without extrathyroidal extension, and without clinical evidence of any lymph node metastases (cN0), the initial surgical procedure can be either a bilateral procedure (near total or total thyroidectomy) or a unilateral procedure (lobectomy). Thyroid lobectomy alone may be sufficient treatment for small $(<1 \mathrm{~cm})$, low-risk, unifocal, intrathyroidal papillary carcinomas in the absence of prior head and neck irradiation or radiologically or clinically involved cervical nodal metastases [9]. Thyroidectomy without prophylactic central neck dissection may be appropriate for small thyroid papillary carcinomas (T1$\mathrm{T} 2$ ), non-invasive, with negative nodes (cN0) and for most follicular carcinomas.

Node dissections must be performed per compartments. Central therapeutic dissection (level VI) must be associated with total thyroidectomy when there are clinically affected nodes. Prophylactic central dissection (ipsilateral or bilateral) should be considered in patients with papillary thyroid carcinoma with clinically unaffected lymph nodes in the central compartment (cN0), with advanced primary tumors (T3 or T4; $>4 \mathrm{~cm}$ or with invasion of neighboring structures) or affected lateral nodes ( $\mathrm{cN} 1 \mathrm{~b})$. Therapeutic lateral dissection of the cervical lateral compartment should be performed in patients with metastatic lymph nodes. Prophylactic lateral dissection is not indicated [9].

In the case of locally advanced thyroid cancer, the surgical treatment will be specific according to the affected anatomical structure, seeking control of the entire macroscopic disease with function preservation. For unresectable or high-risk R1/R2 resections, neoadjuvant therapy with targeted agents may be considered; even the level of evidence is low (case reports).

\section{Recommendations}

Total thyroidectomy is recommended in indicated cases of DTC, mainly in size $>4 \mathrm{~cm}$ (level of evidence: I, grade 
of recommendation: A). All patients should have a preoperative ultrasound evaluation of the central and lateral neck lymph nodes to plan the surgical procedure (level of evidence: III, grade of recommendation: B).

\section{Staging and risk classification}

AJCC/TNM 8th edition staging is used for predicting disease specific survival. Clinical staging is based on inspection/palpation and imaging (ultrasound, CT, PET-CT, etc.) of thyroid gland and regional lymph nodes. Pathologic staging (pTNM) is based on all information used for clinical staging plus histologic examination plus surgeon's description of gross unresected tumor [13]. Tables 2 and 3 show TNM staging classification. Other factors involving risk of persistent or recurrent disease are included in the ATA classification (Table 4).
Table 3 Differentiated thyroid carcinoma TNM staging AJCC UICC 8th edition

\begin{tabular}{lllll}
\hline $\begin{array}{l}\text { When age } \\
\text { at diagnosis } \\
\text { is... }\end{array}$ & And T is... & And N is... & And M is... & $\begin{array}{l}\text { Then the } \\
\text { stage group } \\
\text { is... }\end{array}$ \\
\hline$<55$ years & Any T & Any N & M0 & I \\
& Any T & Any N & M1 & II \\
$\geq 55$ years & T1 & N0/NX & M0 & I \\
& T1 & N1 & M0 & II \\
& T2 & N0/NX & M0 & I \\
& T2 & N1 & M0 & II \\
& T3a/T3b & Any N & M0 & II \\
& T4a & Any N & M0 & III \\
& T4b & Any N & M0 & IVA \\
& Any T & Any N & M1 & IVB \\
\hline
\end{tabular}

Table 2 Differentiated thyroid carcinoma TNM-staging AJCC UICC 8th edition

\begin{tabular}{|c|c|c|}
\hline T Category & \multicolumn{2}{|l|}{ T Criteria } \\
\hline \multicolumn{3}{|c|}{ Definition of primary tumor $(T)$ for papillary and follicular thyroid carcinomas } \\
\hline TX & \multicolumn{2}{|c|}{ Primary tumor cannot be assessed } \\
\hline T0 & \multicolumn{2}{|c|}{ No evidence of primary tumor } \\
\hline $\mathrm{T} 1$ & \multicolumn{2}{|c|}{ Tumor $\leq 2 \mathrm{~cm}$ in greatest dimension limited to the thyroid } \\
\hline T1a & \multicolumn{2}{|c|}{ Tumor $\leq 1 \mathrm{~cm}$ in greatest dimension limited to the thyroid } \\
\hline $\mathrm{T} 1 \mathrm{~b}$ & \multicolumn{2}{|c|}{ Tumor $>1 \mathrm{~cm}$ but $\leq 2 \mathrm{~cm}$ in greatest dimension, limited to the thyroid } \\
\hline $\mathrm{T} 2$ & \multicolumn{2}{|c|}{ Tumor $>2 \mathrm{~cm}$ but $\leq 4 \mathrm{~cm}$ in greatest dimension limited to the thyroid } \\
\hline $\mathrm{T} 3 *$ & \multicolumn{2}{|c|}{ Tumor $>4 \mathrm{~cm}$ limited to the thyroid, or gross extrathyroidal extension invading only strap muscles } \\
\hline T3a* & \multicolumn{2}{|c|}{ Tumor $>4 \mathrm{~cm}$ limited to the thyroid } \\
\hline T3b* & \multicolumn{2}{|c|}{$\begin{array}{l}\text { Gross extrathyroidal extension invading only strap muscles (sternohyoid, sternothyroid, thyrohyoid, or } \\
\text { omohyoid muscles) from a tumor of any size }\end{array}$} \\
\hline $\mathrm{T} 4$ & \multicolumn{2}{|c|}{ Includes gross extrathyroidal extension into major neck structures } \\
\hline $\mathrm{T} 4 \mathrm{a}$ & \multicolumn{2}{|c|}{$\begin{array}{l}\text { Gross extrathyroidal extension invading subcutaneous soft tissues, larynx, trachea, esophagus, or } \\
\text { recurrent laryngeal nerve from a tumor of any size }\end{array}$} \\
\hline $\mathrm{T} 4 \mathrm{~b}$ & \multicolumn{2}{|c|}{$\begin{array}{l}\text { Gross extrathyroidal extension invading prevertebral fascia or encasing carotid artery or mediastinal } \\
\text { vessels from a tumor of any size }\end{array}$} \\
\hline N Category & \multicolumn{2}{|l|}{ N Criteria } \\
\hline \multicolumn{3}{|c|}{ Definition of regional lymph node $(N)$} \\
\hline NX & \multicolumn{2}{|c|}{ Regional lymph nodes cannot be assessed } \\
\hline No & \multicolumn{2}{|c|}{ No evidence of regional lymph nodes metastasis } \\
\hline NOa* & \multicolumn{2}{|c|}{ One or more cytological or histologically confirmed benign lymph node } \\
\hline N0b* & \multicolumn{2}{|c|}{ No radiologic or clinical evidence of locoregional lymph node metastasis } \\
\hline $\mathrm{N} 1 *$ & \multicolumn{2}{|c|}{ Metastasis to regional nodes } \\
\hline N1a* & \multicolumn{2}{|c|}{$\begin{array}{l}\text { Metastasis to level VI or VII (pretracheal, paratracheal, or prelaryngeal/Delphian or upper mediastinal) } \\
\text { lymph nodes. This can be unilateral or bilateral disease }\end{array}$} \\
\hline N1b* & \multicolumn{2}{|c|}{$\begin{array}{l}\text { Metastasis to unilateral, bilateral, or contralateral lateral neck lymph nodes (Levels I, II, III, IV, or V) } \\
\text { or retropharyngeal lymph nodes }\end{array}$} \\
\hline \multicolumn{2}{|l|}{ M Category } & M Criteria \\
\hline \multicolumn{3}{|c|}{ Definition of distant metastasis $(M)$} \\
\hline \multicolumn{2}{|l|}{ M0 } & No distant metastasis \\
\hline \multicolumn{2}{|l|}{ M1 } & Distant metastasis \\
\hline
\end{tabular}


Table 4 ATA risk stratification system to estimate risk of persistent/recurrent disease

Low risk

Papillary thyroid cancer with all of the following present:

No local or distant metastases

All macroscopic tumor has been resected

No invasion of locoregional tissues

Tumor does not have aggressive histology (aggressive histologies include tall cell, insular, columnar cell carcinoma, Hürthle cell carcinoma, follicular thyroid cancer, hobnail variant)

No vascular invasion

No ${ }^{131}$ I uptake outside the thyroid bed on the post-treatment scan, if done

Clinical N0 or $\leq 5$ pathologic N1 micrometastases $(<0.2 \mathrm{~cm} \text { in largest dimension })^{*}$

Intrathyroidal, encapsulated follicular variant of papillary thyroid cancer*

Intrathyroidal, well-differentiated follicular thyroid cancer with capsular invasion and no or minimal ( $<4$ foci) vascular invasion*

Intrathyroidal, papillary microcarcinoma, unifocal or multifocal, including BRAF V600E mutated (if known)*

Intermediate risk

Any of the following present:

Microscopic invasion into the perithyroidal soft tissues

Cervical lymph node metastases or ${ }^{131}$ I avid metastatic foci in the neck on the post-treatment scan done after thyroid remnant ablation

Tumor with aggressive histology or vascular invasion (aggressive histologies include tall cell, insular, columnar cell carcinoma, Hürthle cell carcinoma, follicular thyroid cancer, hobnail variant)

Clinical N1 or $>5$ pathologic N1 with all involved lymph nodes $<3 \mathrm{~cm}$ in largest dimension*

Multifocal papillary thyroid microcarcinoma with extrathyroidal extension and BRAF V600E mutated (if known)*

High risk

Any of the following present:

Macroscopic tumor invasion

Incomplete tumor resection with gross residual disease

Distant metastases

Postoperative serum thyroglobulin suggestive of distant metastases

Pathologic N1 with any metastatic lymph node $\geq 3 \mathrm{~cm}$ in largest dimension*

Follicular thyroid cancer with extensive vascular invasion ( $>4$ foci of vascular invasion)*

Adapted from reference 9. *Proposed modifications, not present in the original 2009 initial risk stratification system. Although not yet proven, it seems reasonable to consider either more careful follow-up or potentially more aggressive therapies for tumors with the highest risk mutational profiles (particularly those with mutational combinations associated with the poorest clinical outcomes). Xing M. Genetic-guided risk assessment and management of thyroid cancer. Endocrinol Metab Clin North Am. 2019;48(1):109-124

\section{Postoperative adjuvant therapy and follow up}

Risk for local and distant recurrence after radical surgery depends mostly on pathological assessment and age and dictates the need for adjuvant treatment and frequency and type of follow up [8]. Thyroid ablation with radioactive iodine ${ }^{131}$ I (RAI) aims to completely eliminate residual remnant thyroid tissue and presumed neoplastic cells thereby reducing the risk of recurrence and probably the mortality rate. In addition, thyroid ablation allows for an earlier detection of recurrence based on serum Tg measurement and eventually on ${ }^{131}$ I whole-body scan (WBS) during follow up [9]. Thyroid-stimulating hormone (TSH) suppressive therapy (serum $\mathrm{TSH}<0.1 \mathrm{mU} / \mathrm{l}$ ) with levothyroxine (LT4) is mandatory in patients with evidence of persistent disease and is given to regulate the postsurgical hypothyroidism and to inhibit the TSH-dependent growth of residual cancer cells [14]. RAI should be given after TSH stimulation by withdrawing LT4 for 4-5 weeks or by administering recombinant human TSH (rhTSH) to increase isotope uptake [15].

Clinical decisions for an adjuvant treatment with RAI should be based on multidisciplinary discussions case by case in centers with sufficient experience and adequate facilities. As a general rule, there is a consensus to offer RAI adjuvant therapy ( $\geq 100 \mathrm{mCi}, 3.7 \mathrm{GBq}$; rhTSH administration or levothyroxine withdrawal) in those patients with high risk of recurrence and should be considered in intermediate-risk patients and low-risk patients, based on TNM stadification, presenting with clinical risk factors. Low doses of radioiodine $(30 \mathrm{mCi}, 1.1 \mathrm{GBq})$ following thyroidectomy seem to be better tolerated than standard doses achieving similar remnant ablation rate [16]. In general, it is not recommended the administration of ablative RAI in patients with DTC smaller than $4 \mathrm{~cm}$ and no evidence of locoregional 
metastasis, but it could be contemplated in some specific scenarios like tumors with aggressive histology or vascular invasion [17]. Patients pT1b-pT2-pT3a, N0 or N1a, may be considered for low dose RAI ablation (30 mCi, 1.11 GBq). Follow up schedule and test to be performed after surgery and consolidation treatment may vary depending on risk for recurrence or persistent disease, histology, and response to RAI. Serum Tg assays and neck US are the cornerstones of DTC follow-up [18].

In patients treated with total thyroidectomy plus RAI remnant ablation, stimulated serum Tg levels $<1 \mathrm{ng} / \mathrm{mL}$ are highly predictive of an excellent response to therapy. When detectable Tg levels emerge in a patient with negative imaging findings, the response to therapy is classified as indeterminate or biochemical incomplete. Uptake on ${ }^{131} \mathrm{I}$ WBS is highly specific (91-100\%) for the presence of thyroid tissue. Fluorodeoxyglucose-positron emission tomography (FDG-PET) is useful for assessing the extent of disease and defining the prognosis especially for aggressive histologies and serum Tg levels above $10 \mathrm{ng} / \mathrm{dL}$. Patients with DTC should be evaluated 6-12 months after primary treatment and follow-up schedule will be adapted on the initial risk of persistent/recurrent disease and responses to therapy [19], as shown in Fig. 1.

\section{Recommendations}

Decision for adjuvant therapy should be made after risk assessment in a multidisciplinary fashion (level of evidence: III, grade of recommendation: B). Ablative RAI should be offered to high-risk patients and considered in intermediaterisk patients (level of evidence: III, grade of recommendation: A).

\section{Treatment of tumor relapse/metastatic DTC}

Locoregional recurrence occurs in up to $20 \%$ of patients, and distant metastases in approximately $10 \%$ at 10 years. Surgery is the preferred therapy for locoregional recurrent disease or low-volume distant metastatic disease.

RAI is currently the first-line treatment for distant metastases that are RAI-avid. Doses of RAI therapy are, at present, controversial, ranging from 100 to $200 \mathrm{mCi}$. Active surveillance may be considered for patients with low-volume disease with no apparent tumor progression and distant from critical structures.

External beam radiotherapy (EBRT) may be indicated for symptomatic bone metastases and central nervous system spread. Evidence of efficacy of EBRT for unresectable locoregional relapses is low and may be considered together with other locoregional treatments such as local ablations.

Up to two-thirds of patients with metastatic or unresectable locoregional disease lose the ability for iodine uptake leading to RAI-refractory metastatic disease, defined as: no uptake of RAI at the initial diagnosis of distant metastases or locoregional recurrence; progressive loosing of RAI uptake after several sessions of RAI therapy; evidence of different foci of distant metastases, some of them with uptake and some others without RAI uptake in the body scan; tumor progression after an adequate RAI treatment even with previous substantial RAI uptake. Other additional criteria that play a role in the definition of RAI-refractory disease are significant uptake in 18FDG-PET, total cumulative doses of RAI over $600 \mathrm{mCi}$, unresectable primary tumors or aggressive DTC histologies, such as poorly differentiated, insular or Hürtle Cell carcinomas [20]. The final decision on the RAI-refractoriness must be taken by multidisciplinary committees and reassessed during the treatment evolution. When a patient with DTC is classified as refractory to RAI, there is no indication for further RAI treatment. No clear recommendation regarding TSH suppression is available in RAI-refractory DTC. Intravenous bisphosphonate (eg, zoledronic acid) or denosumab therapy may be considered for bone metastases.

For clinically progressive or symptomatic RAI-refractory metastatic DTC, not otherwise amenable to local therapies, multikinase inhibitor (MKI) therapy should be considered (Fig. 2). Two randomized, placebo-controlled, phase III trials (DECISION and SELECT) have demonstrated the efficacy of sorafenib [21] and lenvatinib [22], respectively, in these patients. Both drugs were each associated with statistically significant improvement in progression-free survival (PFS) relative to those patients treated with placebo and were approved by regulatory authorities worldwide for RAI-refractory metastatic disease. Although sorafenib and lenvatinib showed an increase in the response rate and PFS versus placebo, they failed to show significant differences in overall survival due to crossover at progression. Currently, there are no clinical or molecular biomarkers that help us to predict the response to these drugs. The differences between these drugs must be taken into account when deciding which is the best treatment option for each patient considering both efficacy and toxicity profile of these drugs. At progression disease, there is no current evidence of efficacy of sequential treatment with MKIs and patients should be considered for clinical trials.

There are several molecular biomarkers, such as mutations in $B R A F$ or fusions in RET/PTC that may predict the response to specific BRAF or RET inhibitors in advanced RAI-refractory DTC. However, current data is available only from phase I/II clinical trials, with no data on the best treatment sequence and no formal approval in this setting. Recently, the European Medicine Agency (EMA) approved the first tumor agnostic therapy for solid tumors directed to NTRK fusions, larotrectinib. Thyroid cancer is the second most frequent tumor with NTRK fusions with an incidence 
Fig. 1 Post-operative management and follow up adapted on the initial risk of persistent/ recurrent disease and responses to therapy

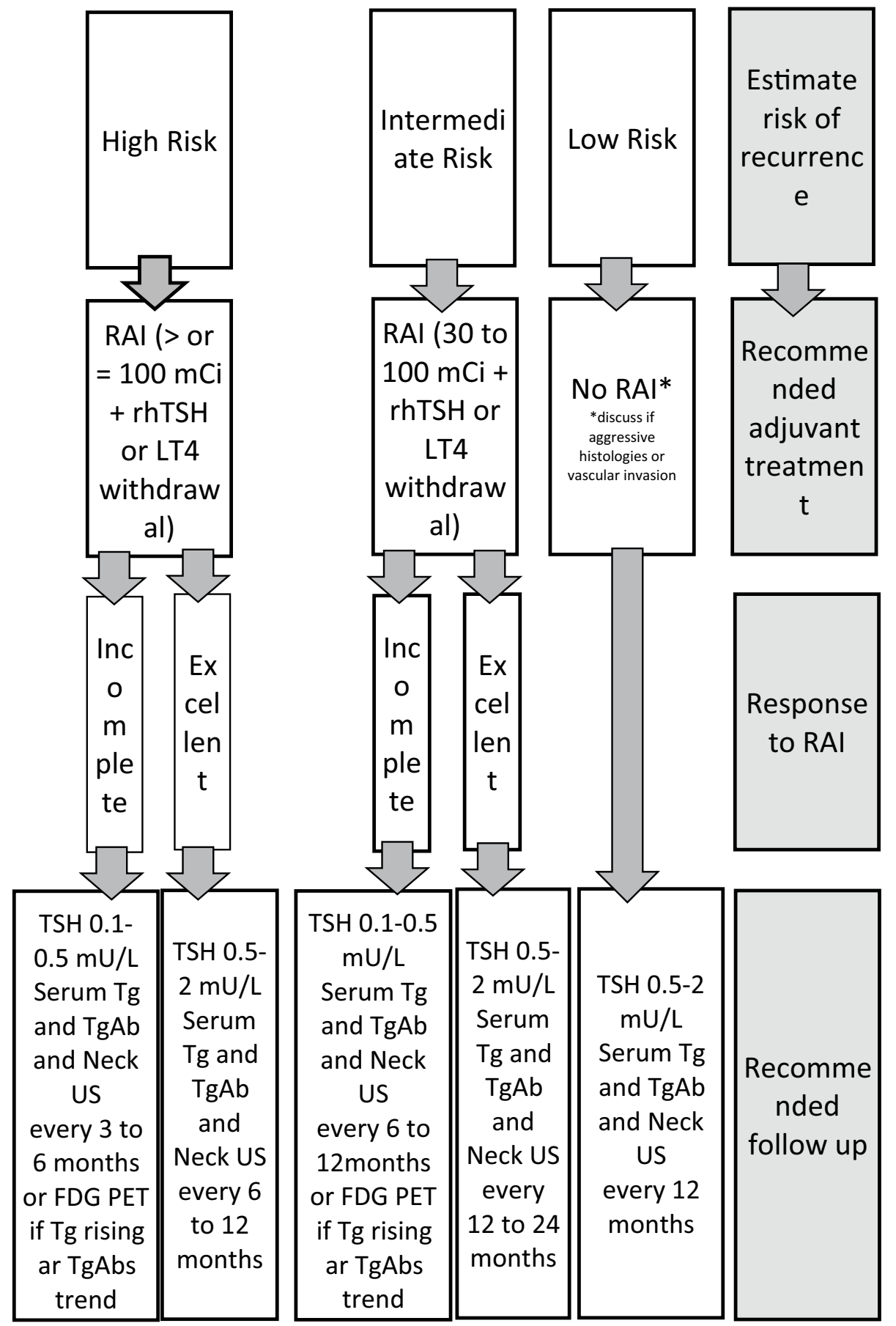

that ranges from 5 to $25 \%$. Larotrectinib demonstrated high durable response rate (including complete responses) in NTRK fusion tumors (79\% of overall response rate in thyroid cancer) and should be considered in the treatment strategy of patients with NTRK altered advanced DTC [23]. Additionally, due to higher target selectivity, toxicity profile is more favorable compared with classical MKIs.

\section{Recommendations}

RAI is the first choice for RAI-avid advanced or relapsed DTC (level of evidence: III, grade of recommendation: B). Lenvatinib or sorafenib should be offered in most patients with RAI-refractory metastatic DTC (level of evidence: I; grade of recommendation: A). 
Fig. 2 Treatment algorithm for advanced RAI-refractory DTC

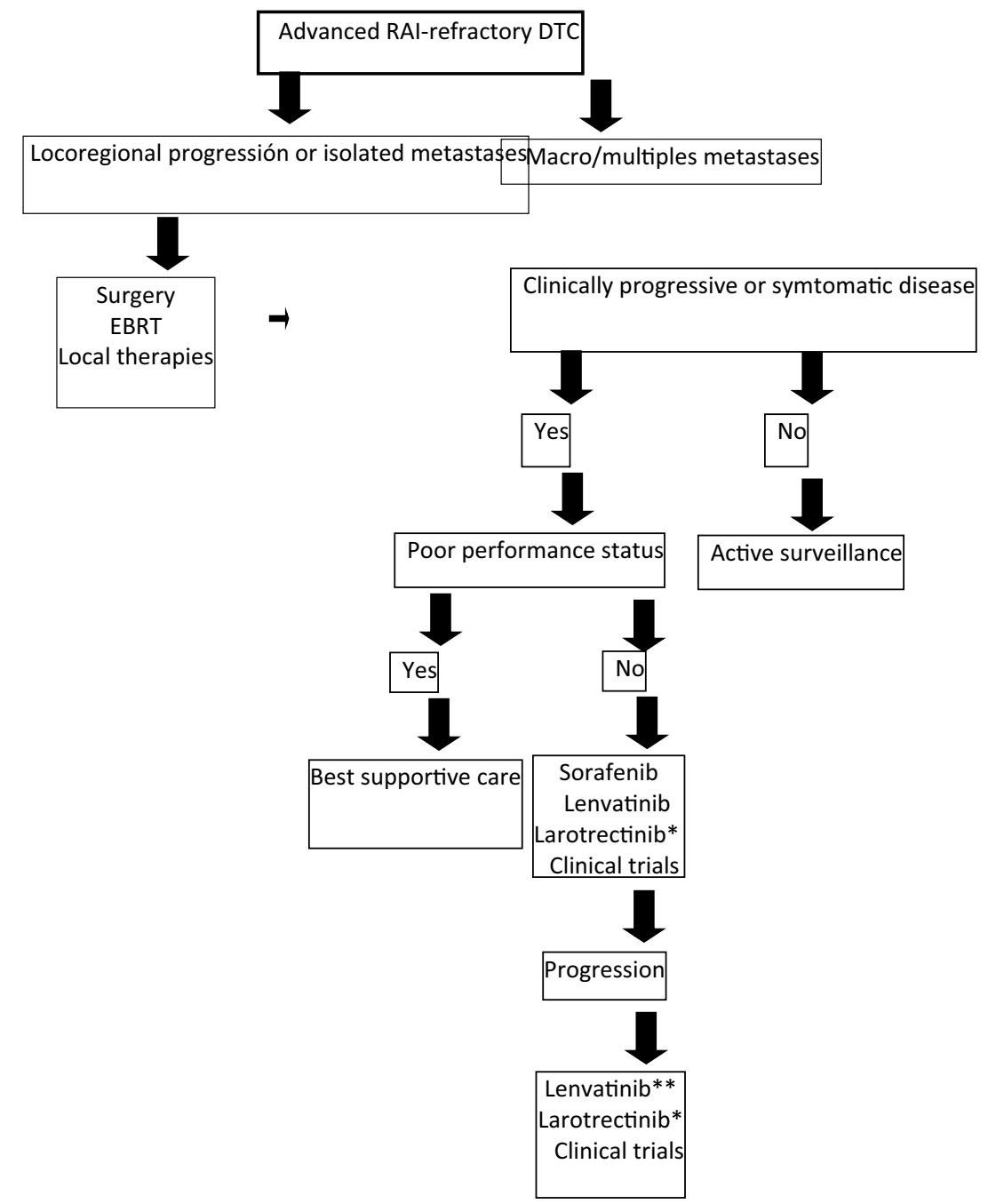

*Larotrectinib: EMA approved (not AEMPS approved yet). Efficacy showed in first-line and after progression to prior MKIs

** Lenvatinib efficacy showed in first and second lines. No data of sorafenib efficacy after lenvatinib

\section{Medullary thyroid carcinoma}

\section{Initial management and follow up}

MTC accounts for 2-8\% of primary thyroid tumors. A thyroid tumor with any suspicious feature of MTC should include immunohistochemistry analysis of calcitonin, chromogranin, carcinoembryonic antigen (CEA) and the absence of Tg. All patients should be offered genetic counseling and be tested for germline RET mutations to rule out the diagnosis of a MEN2 in the family. Preoperative workup includes thyroid and neck ultrasound and basal calcitonin, CEA, calcium, plasma or 24-h urine collection of metanephrines and normetanephrines. Either calcitonin $>500 \mathrm{pg} / \mathrm{mL}$ or signs/ symptoms of regional/distant disease should be studied with contrast-enhanced CT of chest, MRI/ 3-phase CT of liver, axial MRI and bone scintigraphy. Total thyroidectomy and bilateral central neck dissection (level VI) and involved lateral neck compartments (levels II-V) are recommended for neck confined $\geq 1 \mathrm{~cm}$ or bilateral thyroid disease. For unilateral and $<1 \mathrm{~cm}$ tumors, total thyroidectomy should be completed and contralateral neck dissection be considered based on calcitonin levels. Residual lymph node metastases after thyroidectomy, based on calcitonin level, imaging studies or inadequate surgery could benefit from re-operation [24].

Postoperative follow-up includes measurements of calcitonin and CEA levels. Calcitonin $<150 \mathrm{pg} / \mathrm{mL}$ should be evaluated together with physical examination, serum markers and neck ultrasound every 6 months to determine the doubling time of serial measurements and concentrations of 
calcitonin $>150 \mathrm{pg} / \mathrm{mL}$, should be completed with chest CT, MRI or liver three-phase contrast-enhanced CT, bone scintigraphy, pelvis/axial MRI or FDG-PET. Abnormal serum markers in asymptomatic patients with repeated negative imaging can be followed every 6 months or consider reoperation, after excluding liver occult metastasis.

\section{Recommendations}

Total thyroidectomy, bilateral central neck dissection and involved lateral neck compartments are recommended for neck confined $\geq 1 \mathrm{~cm}$ or bilateral thyroid disease for MTC, based on preoperative serum calcitonin levels and neck imaging (level of evidence: III, grade of recommendation: B).

\section{Treatment of tumor relapse/metastatic MTC}

Locoregional recurrence should be approached with compartmental dissection of image-positive or biopsy- positive disease in the central (level VI) or lateral (levels II-V) neck compartments. Postoperative radiotherapy could be considered in case of residual MTC, extrathyroidal extension, or extensive lymph node metastases and risk of airway obstruction, after weighing the benefits against the potential toxicity. Management goals of MTC distant disease are loco-regional disease control, survival increase and the palliation of symptoms. Spinal cord compression and painful bone metastasis require consideration of surgery, EBRT and bisphosphonates. Resection, ablation and liver embolization may be considered in patients with solitary lung and liver metastases.

Somatic RET mutations (30-50\% of sporadic and almost all hereditary MTC), RAS mutations along with overexpression of vascular endothelial growth factor receptor (VEGF) 1 and 2, fibroblast growth factor (FGF) and the plateletderived growth factor (PDGF) are common molecular features in MTC. Based on phase III randomized clinical trials vandetanib and cabozantinib are recommended as systemic therapy in patients with symptomatic or progressive unresectable locally advanced or metastatic MTC. Both vandetanib and cabozantinib demonstrated PFS benefit in front of placebo and a response rate ranging from 28 to $45 \%$ [25, 26]. Treatment decision between both drugs should be based on the different safety profile, baseline patients' characteristics and clinical experience. Beyond the progression on vandetanib or cabozantinib, no other MKI therapy has demonstrated efficacy and treatment on clinical trials should be offered to patients. Based on promising results of clinical research with selective RET inhibitors, patients with $R E T$ mutations should be offered to participate in clinical trials with RET inhibitors both in first-line and in refractory setting after progression to MKIs.
Multidisciplinary approach, experience in dealing with advanced MTC and skills in the management of MKIs are mandatory for the correct management of advanced MTC patients in the daily clinical practice.

\section{Recommendations}

Vandetanib and cabozantinib should be offered as the firstline systemic treatment for progressive locally advanced/ metastatic MTC (level of evidence: I, grade of recommendation: B).

\section{Poorly differentiated thyroid carcinoma}

Poorly differentiated carcinoma is an aggressive thyroid tumor characterized by a partial loss of the features of thyroid differentiation that occupies morphologically and behaviorally an intermediate position between well-differentiated papillary and follicular carcinomas and fully dedifferentiated anaplastic carcinoma. They account for up to $10 \%$ of all thyroid cancers with a 10 -year survival close to $50 \%$ [27].

Diagnostic criteria for poorly differentiated carcinoma are based on the consensus Turin proposal and include the following three features: (i) solid/trabecular/insular microscopic growth pattern, (ii) lack of well-developed nuclear features of papillary carcinoma, and (iii) convoluted nuclei (evidence for partial loss of differentiation in papillary cancer), tumor necrosis, or three or more mitoses per 10 high-power fields [28]. In terms of patient follow-up carcinoma serum $\mathrm{Tg}$ and $\mathrm{TgAb}$ levels should be assessed every 6-12 months and FDG-PET combined with CT is more sensitive than post-therapeutic body scan for detecting persistent disease in these patients [29].

There is no standardized treatment for poorly differentiated thyroid carcinoma to date. However, it is accepted that a more aggressive approach is needed to treat these tumors. If possible, a total thyroidectomy including lymph node dissection should be performed. RAI treatment is only successful in a subset of patients, nevertheless, given the potential therapeutic benefit, high-dose of RAI treatment is currently recommended for all these patients. EBRT for large tumors of $>4 \mathrm{~cm}$ with stage $\mathrm{T} 3$ and $\mathrm{T} 4$ and for patients with regional lymph node metastases should be assessed individually for patients as the data are based on extrapolation from studies of DTC. The basis of systemic treatment in the RAIrefractory setting is MKIs as well as DTC, as this histology subtype was included in registrational phase III studies. Chemotherapy is currently not standard of care, although positive effects have rarely been observed in some patients with platinum- and anthracycline-based schemes [30]. 


\section{Undifferentiated/anaplastic thyroid carcinoma (ATC)}

\section{Initial management and follow up}

Undifferentiated/anaplastic thyroid cancer (ATC) is an aggressive form of thyroid cancer that is associated with a very poor prognosis. Although ATC accounts for $<2 \%$ of all thyroid malignancies, it comprises $>50 \%$ of thyroid cancerrelated mortalities. Overall survival after initial diagnosis is usually less than 6 months with 1-year survival rates of approximately $20 \%$ [31]. The morbidity and poor prognosis of ATC is due either to locoregional disease because of the numerous critical structures located near the thyroid or the high incidence of metastatic disease, with nearly one-half of all patients with ATC presenting with metastatic disease at the time of initial diagnosis. The most common metastatic sites include lungs, bones and brain.

ATC is morphologically heterogeneous and must be distinguished from other neck tumors, including squamous carcinoma of the larynx, sarcomas and lymphomas. Preoperative biopsy assessment includes diagnostic immunomarkers that can differentiate ATC from large cell lymphoma, pleomorphic sarcoma and poorly differentiated DTC. A thorough imaging work-up should be carried out soon after the diagnosis. The FDG-PET scan is the most sensitive tool for documenting the extent of disease. Scans should be repeated at all stages of treatment [32].

\section{Recommendations}

Histological and immunohistochemical accurate study of tumor samples is required to exclude other forms of thyroid cancer. Preoperative radiological studies include ultrasound, $\mathrm{CT}$ or MRI, and FDG-PET scan to rule out distant metastases at initial diagnosis (level of evidence: III, grade of recommendation: $\mathrm{C}$ ).

\section{Treatment of local/locoregional disease}

\section{Surgery}

ATC is rarely amenable to complete resection. Total thyroidectomy with bilateral central neck dissection may be carried out in those very rare cases of localized resectable ATC. Extensive resection with total laryngectomy, esophagectomy and/or resection of the great vessels has been reported in highly selected cases in specialized centers, but postoperative mortality and morbidity are high and there is no high-level evidence to indicate that this approach improves survival. The prognosis is also unaffected by incomplete palliative resection (R2) or 'debulking', which is not generally recommended [33]. Tracheostomy may be needed to alleviate symptoms in patients with moderately progressive disease, but the impact of tracheostomy on quality of life (QoL) should be considered.

\section{Radiotherapy}

Optimal outcomes in terms of survival and local disease control in ATC require complete or near-complete [no residual tumor (R0) or microscopic residual tumor (R1) resection followed by high-dose radiotherapy, with or without concomitant chemotherapy [34]. However, this multimodal approach can strongly impact QoL and should be reserved for carefully selected patients to ensure clinical benefit. Timely discussion within a multidisciplinary team setting is strongly recommended.

A meta-analysis of 17 retrospective studies including 1147 patients looked at the impact of postoperative EBRT after radical ATC resection and found that it significantly reduced the risk of death as compared with radical resection alone (HR 0.556, 95\% CI 0.419-0.737, $P<0.001$ ) [35]. Exploratory analyses demonstrated that EBRT might also confer a survival benefit in patients with stage IVA or IVB disease but not for stage IVC. For best outcomes, EBRT should be delivered as soon as possible after surgery.

Because of the improved dose distribution and the ability to reduce toxicity, intensity-modulated radiotherapy (IMRT) is the recommended approach [36]. There is some evidence of a dose-response relation. An analysis of the United States National Cancer Database showed maximal benefits with doses $>60$ Gy [37].

For stage IVA or IVB disease, concomitant chemotherapy (usually with doxorubicin, platinum agents or taxanes, alone or in combination) has been used. Most of the data reported on this approach came from single-institution series and the clinical benefits reported are variable. In patients with unresectable disease, palliative EBRT has a role in symptom control. The aim is usually to reduce the rate of growth of the neck mass and thereby the pressure symptoms.

Recommendation Total thyroidectomy with lateral and central node dissection should be performed in resectable non-metastatic ATC. Adjuvant EBRT with or without concomitant chemotherapy should be offered to ATC patients with adequate performance status. Timely discussion by a multidisciplinary team is strongly recommended (level of evidence: III, grade of recommendation: B).

\section{Treatment of advanced/metastatic disease}

The priority in ATC patients with distant metastases should be proper control of symptoms, as no randomized study has 
demonstrated benefit in survival or QoL. Novel systemic therapies are urgently needed to improve the generally poor outcomes associated with ATC. Clinical trial enrolment should, therefore, be encouraged for patients with good clinical PS. For patients ineligible for systemic treatments or clinical trials, best supportive care should be discussed.

To date, cytotoxic chemotherapy has been the primary treatment for metastatic disease, but it is associated with very low response rates and significant toxicities. Recommended regimens consist of single-agent therapy with paclitaxel or doxorubicin or combined treatments (e.g., carboplatin/paclitaxel, docetaxel/doxorubicin) administered weekly or every 3-4 weeks $[38,39]$.

No evidence of efficacy of MKIs is currently available in western countries for advanced ATC. An international, multicenter, phase II trial with lenvatinib was stopped early due to futility (NCT02657369).

Molecular profiling studies have begun to elucidate the molecular drivers in ATC [40]. BRAF mutations have been reported up to $40 \%$ of ATC cases. In a phase II, open-label basket trial, patients with $B R A F^{V 600 E}$-positive malignancies (including 16 with ATC) were treated with the BRAF inhibitor dabrafenib (150 mg twice daily) plus the MEK inhibitor trametinib ( $2 \mathrm{mg}$ once daily). The overall response rate was $69 \%(11 / 16)$ and the treatment was well tolerated [41]. In May 2018, this combination received FDA approval for the treatment of locally advanced or metastatic ATC with the $B R A F^{V 600 E}$ mutation. Despite of there were only 16 patients with ATC treated with this combination; the promising results may position this treatment as the first-line therapy for advanced $B R A F^{V 600 E}$ ATC patients. Other rare mutations and genetic aberrations may also prove to be druggable, such as $A L K$ and NTRK translocations. As mentioned before, larotrectinib has the first tumor agnostic approval in Europe for the treatment of NTRK altered cancers. The initial cohort of thyroid cancer patients treated with larotrectinib also included ATC, with objective responses. Extended molecular profiling of ATCs should be strongly encouraged upfront as it may reveal promising possibilities for targeted therapies.

Immunotherapy has reported initial promising activity in patients with advanced ATC regardless $B R A F$ mutations [42]. Additional research is still needed to define the role of immunotherapy in ATC.

\section{Recommendation}

Systemic chemotherapy based on taxanes and/or anthracyclines may be offered in patients with good performance status. New clinical trials may offer an opportunity to improve the outcome in advanced ATC patients. Extended molecular profiling of ATCs should be strongly encouraged upfront and could offer new targeted therapy with higher response chances (dabrafenib-trametinib for $B R A F^{V 600 E}$ mutated and larotrectinib for NTRK fusion ATCs). Clinical trial enrolment should be encouraged (level of evidence: II, grade of recommendation: $\mathrm{C}$ ).

Acknowledgments The guideline was reviewed by Dr. Ismael Capel (Endocrinology Department, Parc Taulí Hospital Universitari. Institut d'Investigació i Innovació Parc Taulí I3PT. Universitat Autònoma de Barcelona. Sabadell, Spain), Dr. Carles Zafon (Endocrinology Department, Hospital Universitari Vall d'Hebron, Barcelona, Spain) and Dr. Joan Castell (Nuclear Medicine Department, Hospital Universitari Vall d'Hebron, Barcelona, Spain). We thank them for their support and valuable contributions.

\section{Compliance with ethical standards}

Conflict of interest Dr. Gallardo reports personal fees and non-financial support from Eisai, personal fees and non-financial support from Bayer, during the conduct of the study; grants, personal fees and non-financial support from Astellas, grants, personal fees and nonfinancial support from Janssen, grants, personal fees and non-financial support from Sanofi, grants, personal fees and non-financial support from Bayer, grants, personal fees and non-financial support from Ipsen, personal fees and non-financial support from Roche, grants from Ferrer, grants from GSK, personal fees from Novartis, personal fees and non-financial support from Pfizer, personal fees and non-financial support from BMS, personal fees from EUSA Pharma, personal fees and non-financial support from Rovi, personal fees and non-financial support from Daiichi Sankyo, personal fees and non-financial support from Leo Pharma, personal fees from Techdow, personal fees and nonfinancial support from Menarini, personal fees and non-financial support from AstraZeneca, personal fees from Boehringer, personal fees from Merck, personal fees from MSD, outside the submitted work. Dr. Medina reports grants, personal fees and non-financial support from Roche, grants, personal fees and non-financial support from Novartis, grants, personal fees and non-financial support from BMS, personal fees and non-financial support from Pierre Fabre, personal fees and non-financial support from MSD, outside the submitted work. Dr. Sánchez reports personal fees (meeting and travel support) from Pierre Fabre and Boehringer Ingelheim; personal fees from Roche; personal fees (advisory board, consultancy, speaker honoraria or travel support) from Eisai, Sanofi, Bristol-Myers Squibb, MSD, Merck Serono and Kyowa Kirin, outside the submitted work. Dr. Viúdez has nothing to disclose. Dr. Grande reports grants from Roche, grants from Pfizer, grants from BMS, grants from IPSEN, grants from EUSA Pharma, grants from MSD, grants from Sanofi, grants from Adacap, grants from Pierre Fabre, grants from Lexicon, grants from MTEM/ THreshold, grants from Astra Zeneca, during the conduct of the study. Dr. Porras reports non-financial support from BMS, personal fees and non-financial support from Pfizer, non-financial support from AstraZeneca, personal fees and non-financial support from Merck, during the conduct of the study. Dr. Ramón y Cajal has nothing to disclose. Dr. Trigo reports personal fees and non-financial support from BMS, personal fees and non-financial support from AstraZeneca, personal fees from Bayer, non-financial support from MSD, personal fees from Takeda, personal fees from Eisai, personal fees from Merck, during the conduct of the study. Dr. Iglesias reports personal fees from Bayer, personal fees from Eisai, during the conduct of the study; personal fees from BMS, personal fees from Merck, personal fees from MSD, personal fees from Roche, personal fees from Sanofi, outside the submitted work. Dr. Capdevila reports grants and personal fees from Bayer, grants and personal fees from Eisai, grants from Astrazeneca, grants and personal fees from Advanced Accelerator Applications, grants and personal fees from Ipsen, personal fees from Novartis, personal 
fees from Pfizer, personal fees from Merck, personal fees from Sanofi, personal fees from Amgen, personal fees from Exelixis, outside the submitted work.

Ethical approval The current study has been performed in accordance with the ethical standards laid down in the 1964 Declaration of Helsinki and its later amendments.

Informed consent For this type of study, informed consent is not required.

Open Access This article is licensed under a Creative Commons Attribution 4.0 International License, which permits use, sharing, adaptation, distribution and reproduction in any medium or format, as long as you give appropriate credit to the original author(s) and the source, provide a link to the Creative Commons licence, and indicate if changes were made. The images or other third party material in this article are included in the article's Creative Commons licence, unless indicated otherwise in a credit line to the material. If material is not included in the article's Creative Commons licence and your intended use is not permitted by statutory regulation or exceeds the permitted use, you will need to obtain permission directly from the copyright holder. To view a copy of this licence, visit http://creativecommons.org/licenses/by/4.0/.

\section{References}

1. Bray F, Ferlay J, Soerjomataram I, Siegel RL, Torre LA, Jemal A. Global cancer statistics 2018: GLOBOCAN estimates of incidence and mortality worldwide for 36 cancers in 185 countries. CA Cancer J Clin. 2018;68(6):394-424.

2. Ferlay J, Colombet M, Soerjomataram I, Dyba T, Randi G, Bettio M, et al. Cancer incidence and mortality patterns in Europe: estimates for 40 countries and 25 major cancers in 2018. Eur J Cancer. 2018;103:356-87.

3. Howlader N, Noone AM, Krapcho M, Miller D, Brest A, Yu M, Ruhl J, Tatalovich Z, Mariotto A, Lewis DR, Chen HS, Feuer EJ, Cronin KA (eds). SEER Cancer Statistics Review, 1975-2016, National Cancer Institute. Bethesda, MD [based on November 2018 SEER data submission, posted to the SEER web site in April 2019, updated 2019 Sep 5, cited 2019 Nov 10]. Available from: https://seer.cancer.gov/csr/1975_2016/

4. Vaccarella S, Franceschi S, Bray F, Wild CP, Plummer M, Dal Maso L. Worldwide thyroid-cancer epidemic? The increasing impact of overdiagnosis. N Engl J Med. 2016;375(7):614-617.

5. Fagin JA, Wells SA Jr. Biologic and clinical perspectives on thyroid cancer. N Engl J Med. 2016;375(11):1054-1067.

6. Brito JP, Gionfriddo MR, Al Nofal A, Boehmer KR, Leppin AL, Reading $\mathrm{C}$, et al. The accuracy of thyroid nodule ultrasound to predict thyroid cancer: systematic review and meta-analysis. J Clin Endocrinol Metab. 2014;99:1253-63.

7. Grant EG, Tessler FN, Hoang JK, Langer JE, Beland MD, Berland LL, et al. Thyroid ultrasound reporting lexicon: white paper of the ACR Thyroid Imaging, Reporting and Data System (TIRADS) Committee. J Am Coll Radiol. 2015;12(12 Pt A):1272-1279.

8. Pacini F, Schlumberger M, Dralle H, Elisei R, Smit JW, Wiersinga W; European Thyroid Cancer Taskforce. European consensus for the management of patients with differentiated thyroid carcinoma of the follicular epithelium. Eur J Endocrinol. 2006;154(6):787-803.

9. Haugen BR, Alexander EK, Bible KC, Doherty GM, Mandel SJ, Nikiforov YE, et al. 2015 American Thyroid Association Management Guidelines for adult patients with thyroid nodules and differentiated thyroid cancer: the American Thyroid Association Guidelines Task Force on Thyroid Nodules and Differentiated Thyroid Cancer. Thyroid. 2016;26(1):1-133.

10. O'Connell K, Yen TW, Quiroz F, Evans DB, Wang TS. The utility of routine preoperative cervical ultrasonography in patients undergoing thyroidectomy for differentiated thyroid cancer. Surgery. 2013;154(4):697-701; discussion 701-3.

11. Nikiforov YE, Seethala RR, Tallini G, Baloch ZW, Basolo F, Thompson LD, et al. Nomenclature revision for encapsulated follicular variant of papillary thyroid carcinoma: a paradigm shift to reduce overtreatment of indolent tumors. JAMA Oncol. 2016;2(8):1023-9.

12. Kuo JH, McManus C, Graves CE, Madani A, Khokhar MT, Huang BJ, et al. Updates in the management of thyroid nodules. Curr Probl Surg. 2019;56(3):103-27.

13. Tuttle RM, Haugen B, Perrier ND. Updated American Joint Committee on cancer/tumor-node-metastasis staging system for differentiated and anaplastic thyroid cancer (eighth edition): what changed and why? Thyroid. 2017;27(6):751-6.

14. McGriff NJ, Csako G, Gourgiotis L, Lori CG, Pucino F, Sarlis NJ. Effects of thyroid hormone suppression therapy on adverse clinical outcomes in thyroid cancer. Ann Med. 2002;34(7-8):554-64.

15. Pacini F, Ladenson PW, Schlumberger M, Driedger A, Luster M, Kloos RT, et al. Radioiodine ablation of thyroid remnants after preparation with recombinant human thyrotropin in differentiated thyroid carcinoma: results of an international, randomized, controlled study. J Clin Endocrinol Metab. 2006;91(3):926-32.

16. Mäenpää HO, Heikkonen J, Vaalavirta L, Tenhunen M, Joensuu $\mathrm{H}$. Low vs. high radioiodine activity to ablate the thyroid after thyroidectomy for cancer: a randomized study. PLoS ONE. 2008;3(4):e1885.

17. Díez JJ, Oleaga A, Álvarez-Escolá C, Martín T, Galofré JC; en representación del Grupo de Trabajo de Cáncer de Tiroides de la Sociedad Española de Endocrinología y Nutrición. [Clinical guideline for management of patients with low risk differentiated thyroid carcinoma]. Endocrinol Nutr. 2015;62(6):e57-72

18. Lamartina L, Grani G, Durante C, Borget I, Filetti S, Schlumberger M. Follow-up of differentiated thyroid cancer-what should (and what should not) be done. Nat Rev Endocrinol. 2018;14(9):538-51.

19. Tuttle RM, Tala H, Shah J, Leboeuf R, Ghossein R, Gonen M, et al. Estimating risk of recurrence in differentiated thyroid cancer after total thyroidectomy and radioactive iodine remnant ablation: using response to therapy variables to modify the initial risk estimates predicted by the new American Thyroid Association staging system. Thyroid. 2010;20(12):1341-9.

20. Capdevila J, Galofre JC, Grande E, Zafón Llopis C, Ramón Y Cajal Asensio T, Navarro González E, et al. Consensus on the management of advanced radioactive iodine-refractory differentiated thyroid cancer on behalf of the Spanish Society of Endocrinology Thyroid Cancer Working Group (GTSEEN) and Spanish Rare Cancer Working Group (GETHI). Clin Transl Oncol. 2017;19(3):279-287.

21. Brose MS, Nutting CM, Jarzab B, Elisei R, Siena S, Bastholt L, et al. Sorafenib in radioactive iodine-refractory, locally advanced or metastatic differentiated thyroid cancer: a randomised, doubleblind, phase 3 trial. Lancet. 2014;384(9940):319-28.

22. Schlumberger M, Tahara M, Wirth LJ, Robinson B, Brose MS, Elisei R, et al. Lenvatinib versus placebo in radioiodine-refractory thyroid cancer. N Engl J Med. 2015;372(7):621-30.

23. Drilon A, Laetsch TW, Kummar S, DuBois SG, Lassen UN, Demetri GD, et al. Efficacy of larotrectinib in TRK fusion-positive cancers in adults and children. N Engl J Med. 2018;378(8):731-9.

24. Filetti S, Durante C, Hartl D, Leboulleux S, Locati LD, Newbold $\mathrm{K}$, et al. Thyroid cancer: ESMO Clinical Practice Guidelines for 
diagnosis, treatment and follow-up. Ann Oncol. 2019. https://doi. org/10.1093/annonc/mdz400.

25. Wells SA, Robinson BG, Gagel RF, Dralle H, Fagin JA, Santoro $\mathrm{M}$, et al. Vandetanib in patients with locally advanced or metastatic medullary thyroid cancer: a randomized, double-blind phase III trial. J Clin Oncol. 2012;30(2):134-41.

26. Schlumberger M, Elisei R, Müller S, Schöffski P, Brose M, Shah $\mathrm{M}$, et al. Overall survival analysis of EXAM, a phase III trial of cabozantinib in patients with radiographically progressive medullary thyroid carcinoma. Ann Oncol. 2017;28(11):2813-9.

27. Asioli S, Erickson LA, Righi A, Jin L, Volante M, Jenkins S, et al. Poorly differentiated carcinoma of the thyroid: validation of the Turin proposal and analysis of IMP3 expression. Mod Pathol. 2010;23:1269-78.

28. Volante M, Collini P, Nikiforov YE, Sakamoto A, Kakudo K, Katoh R, et al. Poorly differentiated thyroid carcinoma: the Turin proposal for the use of uniform diagnostic criteria and an algorithmic diagnostic approach. Am J Surg Pathol. 2007;31(8):1256-64.

29. Nascimento C, Borget I, Al Ghuzlan A, Deandreis D, Hartl D, Lumbroso J, et al. Postoperative fluorine-18-fluorodeoxyglucose positron emission tomography/computed tomography: an important imaging modality in patients with aggressive histology of differentiated thyroid cancer. Thyroid. 2015;25(4):437-44.

30. Yang H, Chen Z, Wu M, Lei T, Yu H, Ge M, et al. Remarkable response in 2 cases of advanced poorly differentiated thyroid carcinoma with liposomal doxorubicin plus cisplatin. Cancer Biol Ther. 2016;17:693-7.

31. Nagaiah G, Hossain A, Mooney CJ, Parmentier J, Remick SC. Anaplastic thyroid cancer: a review of epidemiology, pathogenesis, and treatment. J Oncol. 2011;2011:542358.

32. Bogsrud TV, Karantanis D, Nathan MA, Mullan BP, Wiseman GA, Kasperbauer JL, et al. 18F-FDG PET in the management of patients with anaplastic thyroid carcinoma. Thyroid. 2008;18:713-9.

33. Sugitani I, Onoda N, Ito KI, Suzuki S. Management of anaplastic thyroid carcinoma: the fruits from the ATC research consortium of Japan. J Nippon Med Sch. 2018;85:18-27.

34. Ito K, Hanamura T, Murayama K, Okada T, Watanabe T, Harada $\mathrm{M}$, et al. Multimodality therapeutic outcomes in anaplastic thyroid carcinoma: improved survival in subgroups of patients with localized primary tumors. Head Neck. 2012;34:230-7.
35. Kwon J, Kim BH, Jung HW, Besic N, Sugitani I, Wu HG. The prognostic impacts of postoperative radiotherapy in the patients with resected anaplastic thyroid carcinoma: a systematic review and meta-analysis. Eur J Cancer. 2016;59:34-45.

36. Bhatia A, Rao A, Ang KK, Garden AS, Morrison WH, Rosenthal DI, et al. Anaplastic thyroid cancer: clinical outcomes with conformal radiotherapy. Head Neck. 2010;32:829-36.

37. Pezzi TA, Mohamed ASR, Sheu T, Blanchard P, Sandulache VC, Lai SY, et al. Radiation therapy dose is associated with improved survival for unresected anaplastic thyroid carcinoma: outcomes from the National Cancer Data Base. Cancer. 2017;123:1653-61.

38. Shimaoka K, Schoenfeld DA, DeWys WD, Creech RH, DeConti R. A randomized trial of doxorubicin versus doxorubicin plus cisplatin in patients with advanced thyroid carcinoma. Cancer. 1985;56:2155-60.

39. Ain KB, Egorin MJ, DeSimone PA. Treatment of anaplastic thyroid carcinoma with paclitaxel: phase 2 trial using ninety-six-hour infusion. Collaborative Anaplastic Thyroid Cancer Health Intervention Trials (CATCHIT) Group. Thyroid. 2000;10:587-594.

40. Capdevila J, Mayor R, Mancuso FM, Iglesias C, Caratù G, Matos $\mathrm{I}$, et al. Early evolutionary divergence between papillary and anaplastic thyroid cancers. Ann Oncol. 2018;29(6):1454-60.

41. Subbiah V, Kreitman RJ, Wainberg ZA, Cho JY, Schellens JHM, Soria JC, et al. Dabrafenib and trametinib treatment in patients with locally advanced or metastatic BRAF V600-mutant anaplastic thyroid cancer. J Clin Oncol. 2018;36:7-13.

42. Wirth LJ, Eigendorff E, Capdevila J, Paz-Ares LG, Lin CC, Taylor $\mathrm{MH}$, et al. Phase I/II study of spartalizumab (PDR001), an anti$\mathrm{PD} 1 \mathrm{mAb}$, in patients with anaplastic thyroid cancer. J Clin Oncol. 2018;36(15):6024-6024.

43. Dykewicz Clare A. Summary of the Guidelines for Preventing Opportunistic Infections among Hematopoietic Stem Cell Transplant Recipients. Clinical Infectious Diseases. 2001;33(2):139-44.

Publisher's Note Springer Nature remains neutral with regard to jurisdictional claims in published maps and institutional affiliations. 